\title{
Philonsorbonne
}

1 | 2007

Année 2006-2007

\section{Éthique de la discussion et éthique de la libération : Un dialogue philosophique Nord-Sud}

Fátima HURTADO-LÓPEZ

\section{(2) OpenEdition}

Journals

Édition électronique

URL : https://journals.openedition.org/philonsorbonne/116

DOI : 10.4000/philonsorbonne.116

ISSN : $2270-7336$

Éditeur

Publications de la Sorbonne

\section{Édition imprimée}

Date de publication : 15 novembre 2007

Pagination : 65-83

ISBN : 978-2-85944-594-2

ISSN : 1255-183X

Référence électronique

Fátima HURTADO-LÓPEZ, «Éthique de la discussion et éthique de la libération : Un dialogue philosophique Nord-Sud ", Philonsorbonne [En ligne], 1 | 2007, mis en ligne le 27 janvier 2013, consulté le 08 juin 2021. URL : http://journals.openedition.org/philonsorbonne/116 ; DOI : https://doi.org/ 10.4000/philonsorbonne.116 


\title{
Éthique de la Discussion et Éthique de la Libération : Un dialogue philosophique Nord-Sud
}

\author{
Fátima Hurtado López
}

\section{Introduction}

Le dialogue entre l'éthique de la discussion de Karl-Otto Apel et l'éthique de la libération d'Enrique Dussel commença en 1989 à l'occasion du Séminaire International organisé par Raúl Fornet-Betancourt à Freiburg (Allemagne) intitulé Philosophie der Befreiung. Begründungen von Ethik in Deutschland und Lateinamerika heute («Philosophie de la Libération. Fondations de l'éthique en Allemagne et en Amérique Latine aujourd'hui $\left.»^{1}\right)$. Ce séminaire constitue le début du Programme de Dialogue Philosophique Nord-Sud ${ }^{2}$, celui-ci ayant pour finalité d'impulser le dialogue interculturel en philosophie comme une «méthode » pour le «surpassement réel de la dépendance, l'asymétrie et le colonialisme en vigueur $»^{3}$ dans les domaines économique et politique, mais aussi dans les relations philosophiques entre le Nord et le Sud. L'axe thématique de ce premier Séminaire International était la question de la fondation de l'éthique, cette question étant présentée à la lumière de l'éthique de la discussion du côté Nord, et de la philosophie latino-américaine de la libération du côté Sud. Les deux figures principales de ce premier Séminaire International étaient alors les fondateurs de ces deux modèles philosophiques, à savoir, Karl-Otto Apel d'un côté et Enrique Dussel de l'autre côté. Mais ce premier séminaire est seulement le premier d'une longue succession de rencontres entre ces deux philosophes dans le cadre de ce Programme de Dialogue Philosophique Nord-Sud : 1991 à Mexico, 1992 à Mayence (Allemagne), 1993 à Moscou, puis cette même année à São Leopoldo (Brésil), 1995 à Eichstätt (Allemagne), 1996 à Aachen (Allemagne), 1997 à Mexico, 1998 à El

1. R. Fornet-Betancourt et C. Lértora (edt.), Ethik in Deutschland und Lateinamerika heute, Frankfurt a.M., Peter Lang, 1987.

2. Pour une analyse de l'histoire de ce programme voir R. Fornet-Betancourt, «El programa de diálogo Norte-Sur. Historia de un proceso y balance provisional », in R. Fornet-Betancourt y J..J. Senent (ed.), Filosofía para la convivencia. Caminos de diálogo Norte-Sur, Séville, MAD, 2004, p. 185-194.

3. Ibid., p. 185. 
Salvador, 2001 de nouveau à Mexico ${ }^{4}$. Ces rencontres ont donné à Apel l'occasion de réfléchir de manière explicite aux problèmes du Tiers-Monde, ainsi qu'au défi que l'éthique de la libération représente pour l'éthique de la discussion. De manière symétrique, elles ont fourni un lieu où expliciter la critique que l'éthique de la libération fait de l'éthique apelienne, ainsi que l'occasion d'apprécier le défi que l'éthique de la discussion représente pour l'éthique de la libération. Cependant, si les premiers Séminaires étaient centrés sur la discussion directe entre K.-O Apel et E. Dussel, et dès lors sur les débats à propos de la fondation, la critique et la complémentarité de ces deux modèles philosophiques, une nouvelle étape commence à partir du $\mathrm{V}^{\mathrm{e}}$ Séminaire International du Programme de Dialogue Philosophique NordSud. En effet, à partir de ce moment, le dialogue philosophique s'est trouvé guidé par un "problème réel de notre actualité mondiale », la pauvreté, qui est passé au premier plan des débats : les rencontres n'avaient plus comme finalité le perfectionnement des théories en débat mais la « réalisation de la justice dans l'ordre international $»^{5}$. Les participations et le dialogue entre K.-O. Apel et E. Dussel ont conservé néanmoins une place importante dans ce Programme qui, en raison de son évolution interdisciplinaire, s'est généralisé en un "Programme de Dialogue Nord-Sud», et non plus spécifiquement «philosophique».

Karl-Otto Apel comme Enrique Dussel ont mené leur réflexion philosophique respective à partir des contextes historico-politiques dans lesquels ils se trouvaient. Il est donc important de comprendre dans un premier temps ces différents contextes dans lesquels sont nées l'éthique de la discussion d'un côté et l'éthique de la libération de l'autre côté afin de mieux cerner les différences entre ces deux propositions. Nous allons cependant voir dans un deuxième temps comment, malgré ces racines historicopolitiques différentes, il s'agit chez ces deux auteurs d'un clair pari pour un universalisme authentique qui aille au-delà du solipsisme méthodologique et de l'universalisme totalitaire. Le monde se trouve aujourd'hui face à deux grands problèmes planétaires : les problèmes socio-économiques Nord-Sud d'un côté, et la crise environnementale de l'autre côté. La philosophie doit pouvoir s'approprier ce nouveau défi et répondre aux nouvelles questions d'échelle planétaire qui se posent à l'humanité dans son ensemble. Les propositions de ces auteurs, bien que différentes, s'accordent de ce fait en ce

4. Les six premières rencontres ont été recueillies dans l'ouvrage K.-O. Apel, E. Dussel, Ética del discurso y ética de la liberación, Madrid, ed. Trotta, 2004. La participation d'Apel dans la septième rencontre a été publiée, inédite en espagnol, dans R. Fornet-Betancourt (ed), Armut im Spannungsfeld zwischen Globalisierung und dem Recht auf eigene Kultur, Frankfurt a.M., IKO-Verlag, 1998, p. 106-130. La participation de Dussel dans la septième, huitième et neuvième rencontres ont été publiées dans E. Dussel, Hacia una filosofía política crítica, Bilbao, ed. Desclée, 2001, p. 111-126, 65-87 et 145-157 respectivement. La dernière rencontre correspond au texte de Dussel «Estado de guerra permanente y razón cínica », dans Herramienta (Buenos Aires), VII/21.

5. R. Fornet-Betancourt y J.J. Senent (eds.), Filosofía para la convivencia. Caminos de diálogo Norte-Sur, op. cit., p. 190. 
point : il s'agit chez Apel comme chez Dussel d'une éthique universelle. Cette éthique universelle, si elle veut faire face aux deux grands défis cités plus haut, doit être par ailleurs une éthique de la responsabilité et de la solidarité. Ainsi, nous étudierons dans un troisième temps la question de la responsabilité. Ce point constitue en même temps une ouverture vers de nouveaux débats entre ces deux auteurs. En résumé, il s'agit au cours de ces pages de présenter l'enrichissant dialogue entre ces deux éthiques, en explicitant leurs points forts et leurs points faibles, leurs convergences et leurs divergences, et surtout leur enrichissante, mais en même temps conflictuelle, complémentarité.

\section{Les différents contextes}

L'éthique de la discussion apelienne comme l'éthique de la libération dusselienne sont nées autour des années soixante-dix du siècle dernier. Cependant, si elles partagent le fait d'être apparues dans un moment commun d'instabilité politique, elles proviennent néanmoins de réalités historico-politiques très différentes. Ainsi, l'expérience de K.-O. Apel est celle d'un citoyen allemand né à Düsseldorf, en 1922. Il appartenait ainsi à une génération qui avait vécu la catastrophe du national-socialisme, expérience qui dans le cas de cet auteur signifiait la «destruction de la conscience morale». E. Dussel, de son côté, est né dans « un village duquel García Márquez aurait pu écrire de nouveau Cent ans de solitude ${ }^{\circ}: \mathrm{La} \mathrm{Paz}$ (Colocorto à l'époque coloniale), en Argentine, en 1934. L'éthique de la libération, qu'il développa presque à la même période que l'éthique de la discussion apelienne, avait comme provocation centrale l'expérience historique de dépendance et de sous-développement vécue en Amérique Latine. Si la différence géographique ne doit pas être réduite de manière relativiste - ce qui bloquerait le dialogue et la possible complémentarité entre ces deux éthiques -, elle nous offre cependant les racines à partir desquelles mûriront les principaux intérêts qui caractériseront les deux propositions étudiées. Voyons dès lors comment ces différents contextes ont pu être à l'origine de chacune des éthiques.

\section{L'éthique de la discussion comme une éthique à l'époque de la science et de la technique}

Son expérience dans l'armée allemande provoqua chez Apel sa première inquiétude philosophique. Cette inquiétude fut motivée par son désir de

6. Enrique Dussel, «Un proyecto ético y político para América Latina », Anthropos, $\mathrm{n}^{\circ} 180$ (sept.-oct. 1998), p. 14. La traduction est nôtre. 
comprendre comment il avait été possible dans le Troisième Reich que tout un peuple s'acquitte de ses obligations envers la nation, et cela malgré le fait de comprendre que le peuple servait - avec des conséquences effroyables un régime «profondément immoral». Apel essayait d'apprécier, dans la catastrophe nationale qu'il était conscient de vivre, des aspects pourtant significatifs du point de vue moral. Il a ainsi commencé la reconstruction du développement de la conscience morale, dans un effort pour découvrir, à partir de ce point de vue spécifiquement allemand, des critères néanmoins universellement valides.

À partir de l'hiver de 1945-46, le jeune Apel, de retour de la guerre et avec une immense soif d'information, commença ses études d'histoire politique, d'histoire des idées et, finalement, de philosophie. Néanmoins, si cet emplacement géographique et historique influence dans une certaine mesure le point de départ de la philosophie d'Apel, il reste finalement secondaire dans son éthique de la discussion et, même s'il a une certaine importance, l'éthique de la discussion ne peut pas «en aucune façon [...] être réduite à lui [à cet emplacement géographique] de manière relativiste ${ }^{7}$. En revanche, au-delà de cette problématique interne allemande, les questions centrales de l'inquiétude philosophique d'Apel seront la transformation de la philosophie transcendantale kantienne et le problème d'une fondation rationnelle de la validité intersubjective de l'éthique universelle et planétaire dont l'humanité, dans une époque de progrès technique et scientifique, semble plus que jamais avoir besoin.

Apel a commencé par expliquer la conception d'une reconstruction du passage - encore inaccompli - à la morale post-conventionnelle ${ }^{8}$ et, dès lors, universelle. En effet, son expérience de la guerre, mais aussi sa conscience de la crise environnementale et du problème alimentaire, lui feront apprécier dans ces problèmes posés à l'humanité dans son ensemble un défi moral inéluctable. Apel sent le besoin de trouver - pour une survie véritablement

7. K.-O. Apel, E. Dussel, Ética del discurso y ética de la liberación, Madrid, ed. Trotta, 2004, p. 251. Les traductions que nous incluons dans ce travail sont nôtres.

8. Les morales «conventionnelles » de Kolhberg sont celles qui correspondent aux stades 3 et $4 \mathrm{du}$ développement de la conscience morale. Il s'agit des morales traditionnelles des « usages ». Le stade 3 est celui où la loyauté n'est exigible que dans le cadre des rapports interhumains impliqués par des liens de sang. Le stade 4 est celui de la loi et de l'ordre, des devoirs sont acquis vis-à-vis de l'État et intériorisés en tant que tels. La morale conventionnelle est donc la morale interne correspondant aux systèmes d'auto-affirmation que sont les États. En revanche, la morale post-conventionnelle est une morale universaliste qui relève des principes dictés par la raison et de la conscience morale personnelle. Le stade 5 post-conventionnel est «celui de l'orientation contractuelle légaliste avec une connotation utilitariste ». Le dernier stade de la logique de développement de la conscience morale de Kohlberg, le stade 6 post-conventionnel, est le stade de la « conscience morale autonome qui s'oriente en fonction du principe de la réciprocité strictement généralisée ». Selon Apel, le passage d'une morale conventionnelle à une morale post-conventionnelle est nécessaire pour que puisse apparaître une éthique universaliste de la raison, une macro-éthique à l'échelle planétaire. Pour ces distinctions, voir entre autres K.-O. Apel, Discussion et responsabilité, vol. 2, op. cit., p. 17-20. 
humaine de l'humanité - une fondation philosophique ultime des principes moraux universels ${ }^{9}$.

Cependant, Apel constate que cette question débouche sur une situation paradoxale. D'une part, «la nécessité d'une éthique de la responsabilité solidaire ayant une force d'obligation intersubjective et engageant toute l'humanité quant aux conséquences que peuvent engendrer les activités et des conflits humains, n'a jamais été aussi urgente qu'aujourd'hui [...]. D'autre part, la fondation rationnelle d'une éthique intersubjectivement valide n'a apparemment jamais été aussi difficile qu'actuellement ; la raison en est que la science moderne s'est emparée la première du concept de fondation rationnelle, intersubjectivement valide [...]. Dans ces conditions précisément, une éthique rationnelle de réduction des conflits semble impossible $»^{10}$. De ce fait, à notre époque, à l'ère de la science, fonder en raison une éthique universellement valide paraît donc paradoxalement à la fois nécessaire et impossible. Nécessaire car la co-existence des nombreuses et diverses formes de vie a besoin des normes d'une éthique universelle capable d'organiser une co-responsabilité solidaire (cette macro-éthique ou éthique globale doit être en même temps susceptible d'organiser la coresponsabilité quant aux conséquences engendrées par des activités scientifiques et techniques collectives, que ne peut pas supporter l'individu seul). Impossible car la conception scientiste de la science l'empêche : si la science moderne s'empare du concept de fondation rationnelle, toutes les élaborations théoriques qui ne sont pas axiologiquement neutres deviennent de pures et simples idéologies, et l'éthique, les valeurs ultimes et les normes, deviennent dès lors des décisions en conscience, c'est-à-dire irrationnelles.

Le plus grand défi de ce philosophe sera donc ce fundamentum inconcussum rationnel et objectif de l'éthique. Son principal adversaire sera de ce fait le scepticisme moral pour qui la validité des énoncés normatifs recèle quelque chose de purement subjectif.

\section{L'éthique de la libération comme une éthique dans le contexte de la dépendance et du sous-développement}

Dans l'intervention de Dussel lors de la première rencontre célébrée à Freiburg le 25 novembre 1989, Dussel propose un texte analogue au Zurück zur Normalität? d'Apel. L'auteur réfléchit dans cette première rencontre à l'origine de la philosophie de la libération ainsi qu'aux différences dues aux contextes historiques qu'on peut trouver entre ces deux auteurs en ce qui concerne la perspective philosophique.

9. Voir A. Cortina, Razón comunicativa y responsabilidad solidaria, Salamanca, ed. Sígueme, 1988, p. 16.

10. K.-O. Apel, Discussion et Responsabilité, vol. 1, Paris, éd. du Cerf, 1996, p. 134. 
En effet, la philosophie de la libération, bien que née dans les années soixante du $\mathrm{XX}^{\mathrm{e}}$ siècle, c'est-à-dire presque en même temps que l'éthique de la discussion apelienne, se situe cependant dans l'autre côté du monde, fait qui déterminera d'une manière importante ses inquiétudes et ses catégories philosophiques. Depuis le début l'éthique de la libération s'est présentée comme une éthique dans le contexte de la dépendance, de l'échec du « développementisme ${ }^{11}$ et de la misère des peuples du Sud. L'éthique de la libération tient compte des différents types d'exclusion, ainsi que du problème environnemental auquel se voit confrontée l'humanité de nos jours. Cependant, ces problématiques restent secondaires à côté de la tâche fondamentale de la philosophie de la libération : l'élaboration, à partir de l'exclusion des peuples du Sud, d'une philosophie latino-américaine authentique face à la pensée imitative et eurocentrique, victime et collaboratrice de l'oppression. Pour ce faire, Dussel s'appuie au niveau théorique sur la «théorie de la dépendance » et sa thématique face à l'échec du développementisme. La «théorie de la dépendance» a dû affronter de nombreuses critiques. Cependant, elle a le mérite d'avoir cassé l'enchantement de la «tromperie développementiste», d'avoir exhibé l'articulation structurale des nations sous-développées au système mondial, ce qui constitue d'après la philosophie de la libération la cause externe de la misère des pays sous-développés. Cette expérience d'appartenir à la partie exploitée du monde capitaliste s'est imposée philosophiquement à Dussel comme «l'exigence de surpasser cette "ontologie" ${ }^{12}$.

La découverte d'Emmanuel Levinas en 1969 offre de nouvelles catégories philosophiques qui seront bien reçues par l'éthique de la libération. Ainsi, la catégorie d'Autrui permettait à la philosophie de la libération de mieux indiquer cette «expérience originaire » qui consistait en la prise de conscience du «fait » massif de la domination - domination du Nord qui conditionne, suivant la théorie de la dépendance, le sousdéveloppement du Sud. Le dépassement de l'ontologie qu'essayait l'éthique politique de Dussel, tenant comme point de départ la misère du peuple latino-américain, fut donc possible grâce aux catégories empruntées à l'œuvre levinasienne Totalité et Infini: la catégorie méta-physique ${ }^{13}$ d'Autrui présentait pour la philosophie de la libération la possibilité d'une «trans-ontologie ${ }^{14}$, la notion d' « extériorité » exprimait cette « expérience originaire » vécue par Dussel en tant qu'habitant de la moitié Sud du monde.

11. Le mot espagnol « desarrollismo » - ou « falacia desarrollista »- est difficile à traduire. Il s'agit de la position selon laquelle le chemin modernisateur est évidement celui déjà parcouru par la culture qui s'autoconsidère comme la plus développée, c'est-à-dire que le développement qu'a suivi l'Europe devra être suivi unilinéairement par toute autre culture.

12. K.-O. Apel, E. Dussel, Ética del discurso y ética de la liberación, p. 79.

13. Chez Dussel la notion de «méta-physique » ne renvoie pas à une réalité transcendante à ce monde, mais à l'autre en tant qu'Autre, Autrui, qui se trouve dans l'Extériorité, au-delà de l'être et de la Totalité du système.

14. La proposition de Dussel comporte un projet ontologique de libération déployé depuis l'au-delà du système en vigueur, dans l'Extériorité, et en ce sens trans-ontologique. 
Le point de départ de l'éthique de la libération est donc l'exclusion et la domination comme "négation de l'extériorité constitutive et historique d'Autrui ».

Avec ces outils philosophiques, Dussel pénètre plus avant dans la critique radicale de la prétention universaliste de la raison européenne et la critique des structures d'oppression constatées dans la «communauté réelle de communication». Dans son choix pour les pauvres, la question de la libération des peuples du Tiers-Monde devient le thème central de cette philosophie ; une libération qui se présente à différents niveaux : féministe, pédagogique, politique, économique, etc. Son principal adversaire sera de ce fait le cynique, celui qui nie la priorité de la raison discursive, celui qui nie toute rencontre argumentative. Le «cynisme» est, d'après la définition qu'en donne Dussel ${ }^{15}$, «l'affirmation de la Puissance du système comme fondement d'une raison qui contrôle ou gouverne la raison stratégique comme médiation de sa propre réalisation (comme Puissance absolue) ». Le moment premier de la «raison cynique » est l'affirmation du Tout, sans Altérité ; c'est la négation d'Autrui depuis le début.

\section{Deux approches pour une éthique universaliste}

Nous expérimentons d'une manière spéciale de nos jours le besoin de passer des morales conventionnelles - pré-universalistes et internes aux systèmes sociaux d'auto-affirmation - à une morale post-conventionnelle et dès lors universaliste. Ce besoin particulièrement urgent pour notre époque trouve son explication dans le fait que l'humanité actuelle se voit pour la première fois confrontée à des conflits dont l'envergure dépasse largement les frontières des États, jusqu'à devenir des conflits à échelle planétaire. Dans ce contexte, les morales traditionnelles, préoccupées de garantir la sécurité interne des nations, demeurent insuffisantes. Aujourd'hui, nous avons besoin d'une éthique capable d'une part de rendre possible la coexistence et la coopération responsable des diverses formes de vie à l'intérieur comme à l'extérieur des frontières nationales, et d'autre part - et étant donné la «crise écologique » dont nous sommes les premiers, à l'époque contemporaine, à prendre conscience - capable aussi d'organiser ce qu'Apel nomme une « organisation communicationnelle de la responsabilité collective eu égard aux conséquences que pourront avoir les activités collectives - science et technique ». Ces deux grands problèmes planétaires que vit l'humanité de nos jours - celui concernant les relations interculturelles et internationales équitables et celui concernant les

15. E. Dussel, « Del escéptico al cínico. Del oponente de la ética del discurso al de la filosofía de la liberación », in K.-O. Apel, E. Dussel, Ética del discurso y ética de la liberación, op. cit., p. 171-181. Ce texte correspond à la participation de Dussel lors de la troisième rencontre célébrée à Mayence (Allemagne) le 12 avril 1992. 
conséquences planétaires et écologiquement catastrophiques des sociétés industrielles contemporaines - sont deux problèmes qui, dans les termes d'Apel, se posent aujourd'hui pour la première fois « à l'échelle planétaire comme le problème d'une macroéthique universaliste aux dimensions de l'humanité " ${ }^{16}$.

Cette macroéthique universaliste n'est pas possible avec les présupposés du système occidental de complémentarité qui maintiennent le dualisme entre, d'un côté, une raison instrumentale et axiologiquement neutre, propre à la science et à la technique et, d'un autre côté, et pour tout ce qui concerne les valeurs ultimes et les normes, une décision en conscience, c'est-à-dire irrationnelle. Par conséquent, le problème de la possibilité d'une rationalité éthique ou d'une fondation rationnelle des normes intersubjectivement valides constituera une question capitale dans la réflexion concernant une macroéthique universaliste. Si l'intérêt objectif de la raison théorique est la vérité et la connaissance, l'intérêt objectif de la raison pratique consiste à élucider les intérêts individuels ou subjectifs qui peuvent être proposés par chaque membre de la communauté comme des exigences. Apel posera la nécessité de cette nouvelle fondation de l'éthique sur les bases d'une philosophie transcendantale transformée, en effectuant ainsi le passage du sujet monologique au «nous» de la communauté de communication. Ce passage permet le dépassement du solipsisme méthodologique moderne au nom de l'a priori de la communauté de communication.

L'éthique de la discussion n'offre pas de norme concrète de contenu, mais elle signale les conditions formelles requises afin qu'une norme puisse obliger avec légitimité, et ce de manière universelle. Le philosophe de Düsseldorf affirme en ce sens que «seule la rationalité propre à la communication consensuelle présuppose des règles ou des normes qui se situent a priori au-delà de l'intérêt particulier individuel ${ }^{17}$, ce qui peut alors constituer la base d'une éthique universaliste. Aux yeux d'Apel, la possibilité de fonder en raison l'éthique résulte du fait de prendre conscience du caractère incontournable de la discussion argumentée (en ce sens que celui qui désire l'éliminer rationnellement se voit obligé de faire appel à des arguments, ce qui constitue une contradiction performative), et donc aussi des conditions de possibilité normatives et éthiques requises par l'argumentation. L'argumentation rationnelle, si elle veut atteindre sa propre fin et avoir de ce fait du sens, présuppose la validité de normes éthiques universelles que celui qui argumente a dû accepter depuis toujours.

L'éthique de la discussion apelienne découvre alors la norme morale fondamentale qui oblige celui qui désire argumenter avec sens, à accepter depuis toujours et comme un impératif catégorique, la reconnaissance réciproque de tous les membres de la communauté, qui possèdent tous les mêmes droits et sont également obligés à accomplir les normes basiques

16. K.-O. Apel, Discussion et responsabilité, vol. 1, op. cit., p. 11. Les italiques sont nôtres.

17. Ibid., p. 30-31. 
d'une discussion argumentée. De ce fait, d'après cette norme morale fondamentale, exclure ou nier certains interlocuteurs réels ou virtuels, ne pas les reconnaître, limiter leur capacité argumentative, mépriser les intérêts d'éventuelles personnes affectées des conséquences qui, très certainement, surgiront de l'accomplissement universel d'une norme, ou dialoguer en situation d'inégalité, tout ceci suppose détruire la fin propre de l'argumentation. L'a priori de la communauté de communication constitue une question-clé car elle exige de manière catégorique l'accomplissement de certaines normes morales formelles universelles. Les discussions pratiques permettent également de discerner à travers l'argumentation si une norme est intersubjectivement valide ou non. L'éthique de la discussion est donc une éthique «à deux niveaux, consistant dans une procédure formelle de fondation ultime qui, à son tour, fonde la justification, par le moyen d'une communication consensuelle, des normes concrètes ${ }^{18}$.

Dussel ne nie pas la position d'Apel, mais son point de vue se situe dans une perspective différente. D'après cet auteur, le point de départ ne peut pas se trouver dans l'argumentation, dans la «communauté de communication » d'Apel puisque, comme le montre la réalité historique, plus de $75 \%$ de la population du globe est exclue des discussions : si empiriquement il n'existe pas de symétrie, ni de justice dans la communauté réelle de communication, on ne peut alors atteindre la validité pratique cherchée. Apel avoue lui-même que c'est dans cette question de l'application que l'éthique de la discussion rencontre ses limites, car comme l'auteur le reconnaît à plusieurs reprises, dans une communauté communicative réelle et historiquement conditionnée, « on ne peut ni ne doit nullement partir de la supposition que les conditions d'application de l'éthique de la communication sont déjà réalisées ${ }^{19}$. Le philosophe de l'éthique de la libération part néanmoins de cette limite et situe le point de départ éthique dans le fait massif des «besoins basiques » des majorités misérables. La philosophie qui affirme le «nous» et la « communauté de communication » n'est pas suffisante si l'on se situe de l'autre côté du monde, le côté qui ne peut pas participer car il demeure exclu de l'argumentation et de la vie. Dussel est conscient de l'importance et de la créativité de la pensée d'Apel, mais en même temps, il la considère insuffisante car, en tant que morale formelle de la validité intersubjective, elle n'a pas su intégrer le moment matériel de vérité pratique, ce qui, aux yeux du philosophe argentin, est indispensable surtout à partir du moment où les conséquences du système, qui provoque inévitablement des victimes, deviennent intolérables. De ce fait, l'universalisme de Dussel aura comme principale caractéristique et différence face à l'universalisme formel de l'éthique de la discussion, le fait de revaloriser et de rendre prioritaire l'aspect matériel de l'éthique. En ce sens, et pour l'éthique de la libération, la reproduction et le développement de la vie du sujet humain constitueront, à la différence de l'éthique de la discussion, le critère fondamental de vérité

18. Ibid., p. 105.

19. K.-O. Apel, Discussion et responsabilité, vol. 2, op. cit., p. 77. 
(théorique et pratique). Cet aspect du «contenu » de l'éthique détient une universalité propre et détermine matériellement la morale formelle, il représente le critère universel de "vérité » d'une norme selon qu'elle constitue ou non une médiation pour la vie du sujet. Mais l'universalisme de Dussel n'est pas seulement matériel. En effet, si ce moment matériel ou de contenu de l'éthique est en rapport avec la vérité pratique, le moment formel va se référer à la validité morale. Ces deux moments se co-déterminent et sont dès lors, d'après Dussel, nécessaires tous les deux car le pauvre se trouve en même temps matériellement opprimé et formellement exclu. De sorte que l'universalisme de Dussel, à la différence de celui d'Apel, inclut un moment préalable de contenu, qui s'exprime sous la forme d'un principe matériel éthique universel.

Après la huitième rencontre, lors du Colloque célébré à Mexico en 1997, Dussel présente dans un petit travail intitulé « Principes, médiations et le "bien" comme synthèse ${ }^{20}$ le résumé de l'architectonique de l'éthique de la libération qui avait été élaborée «avec Apel, au-delà d'Apel »1. Au Niveau $\mathrm{A}^{22}$ ou $d^{\prime}$ ' universalisation » abstraite, Dussel énonce les trois principes universels qui fondent l'universalisme de l'éthique de la libération. Le principe de validité discursive ou principe de la morale formelle récupère la transformation discursive du kantisme : une action sera valide si elle est décidée grâce à la participation, rationnelle et symétrique, de tous les concernés dans une communauté de communication. Mais, comme nous l'avons vu, c'est ici que l'éthique de la discussion avoue ses limites car, empiriquement il n'y a pas de symétrie dans la communauté réelle de communication. Dussel introduit alors le principe matériel universel ou de vérité pratique, qui lui sera antérieur. Ce principe est proposé contre les formalismes mais aussi contre les éthiques matérielles antérieures qu'il considère particularistes. Si la vie humaine - qui inclut non seulement la survie biologique, mais aussi la plénitude culturelle et la dignité intégrale est le mode-de-réalité (Realitätsmodus) de l'être humain et le critère de vérité pratique, le principe éthique matériel universel consiste en l'obligation éthique de "produire, reproduire et développer la vie humaine en communauté », et, en dernière instance, de toute l'humanité, c'est-à-dire avec une prétention de vérité pratique universelle. Dussel introduit en troisième lieu un principe universel de faisabilité, qui énonce l'obligation éthique d'exécuter une action qui soit «possible» du point de vue empirique, technique, économique, politique et historique, à l'intérieur du cadre des deux principes antérieurs. Ces trois principes ont leurs contreparties « critiques » définies à partir des effets négatifs car, à partir du

20. «Principios, mediaciones y el "bien" como síntesis (de la "ética del discurso" a la "ética de la liberación") », dans E. Dussel, Hacia una filosofía política crítica, Bilbao, éd. Desclée, 2001, p. 65-85. La traduction est nôtre.

21. Pour une explication détaillée de cette architectonique voir E. Dussel, Ética de la Liberación en la edad de la globalización y de la exclusión, Madrid, ed. Trotta, 1998.

22. De manière analogique à la Teil A d'Apel. 
moment où les effets d'une institution ou d'un système sont considérés comme insupportables, le projet du système dominant devient insoutenable et illégitime pour la victime, qui le juge comme «cause » de l'injustice dont elle souffre. C'est le moment de «l'éthique critique» et Dussel propose alors la version négative des trois principes antérieurs. Au niveau du « sujet de vie » qui ne peut pas vivre, le principe matériel universel critique pose le devoir de critiquer tout système institutionnel qui empêche les victimes (les membres potentiels niés, les exclus, etc.) de vivre. Au niveau du sujet de la rationalité qui ne peut participer ni à l'argumentation ni à la discussion à propos de la reproduction de sa vie, le principe critique de validité ou principe discursif critique énonce l'organisation symétrique des victimes dans une communauté de vie et de communication qui ont pour objectif la critique du système qui les avait niées mais aussi - positivement - la projection d'une alternative future de transformation. Finalement, et au niveau du «sujet de l'action » qui doit transformer les institutions qui le victimisent, le principe de faisabilité critique ou Principe-Libération énonce, en conclusion des principes précédents, le devoir, de la part de la communauté critique, de dé-construire effectivement dans le système sa négativité et de transformer le système afin que les victimes puissent vivre et participer symétriquement dans les décisions.

De ce fait, l'essentiel pour l'éthique de la libération ne sera pas le «nous » de la communauté de communication, mais le «vous», «Autrui » ou l'extériorité transcendantale à toute communauté et ontologie. Dans cette perspective, ce qui est d'abord urgent, ce n'est pas tant de décrire les conditions de possibilité de toute argumentation, que de rendre possible l'effectif pouvoir participer dans les discussions, d'effectuer le passage d'exclu à participant. Ce processus de libération est d'après Dussel - et comme on aura le temps de voir dans les paragraphes suivants - antérieur à la communauté de communication d'Apel et constitue le thème central de sa proposition, et une des questions-clés dans le débat entre ces deux auteurs.

Le principal adversaire de l' «universalisme authentique » que cherche Dussel est, à la différence d'Apel, non pas le solipsisme méthodologique, mais l'universalisme totalitaire, car l'éthique de la libération part du soupçon selon lequel la «communauté de communication "réelle" peut toujours être une société hégémonique qui, quand elle domine les cultures, nations, classes, sexes (le féminin), âges (la jeunesse), impose comme "réelle" (et même comme "idéale", ce qui est sa projection en tant qu'idée régulatrice) sa propre particularité ${ }^{23}$. Dans ce contexte, et tenant compte de cette réalité de domination matérielle et d'exclusion formelle dont souffre la plus grande part de l'humanité, le point de départ ou «source originelle » de toute discussion possible devra se trouver, nous dira Dussel, dans l'interpellation ${ }^{24}$

23. K.-O. Apel, E. Dussel, Ética del discurso y ética de la liberación, op. cit., p. 104.

24. Dussel donne un sens spécifique, différent de l'habituel, aux mots «interpellation» et «interpeller» (se trouver face à quelqu'un et lui demander justice par l'accomplissement d'une responsabilité ou devoir contracté). Du latin «interpellare »: c'est un «appeler» 
qu'Autrui lance à la communauté réelle de communication à partir de l'Extériorité : «Autrui » comme la condition a priori de possibilité de toute argumentation possible. Avant d'être "affecté » des accords - nous dira Dussel -, Autrui est exclu des discussions par lesquelles s'obtiendront ces accords. Dès lors, la «condition absolue transcendantale de possibilité » de toute argumentation ne peut être que la re-connaissance d'Autrui en tant que personne, qui à travers son interpellation exige pouvoir, dans un futur, participer («faire partie ») à la «communauté de communication historicopossible ». L'interpellation du pauvre met en question l'accord établi et renouvelle à partir de sa raison critique l'universalisme monologique et totalitaire dans le sens d'une nouvelle et plus authentique universalité.

Néanmoins, Apel reconnaît à plusieurs reprises l'inapplicabilité immédiate de la norme fondamentale de l'éthique de la discussion exigeant un règlement des conflits par la formation du consensus entre les parties concernées, et ce par la pure voie de la discussion. Notre point de vue est que plus qu'une opposition radicale entre ces deux éthiques, on peut en revanche découvrir une relation de complémentarité et de possible parallélisme, tout en reconnaissant les différents accents dépendants des différents contextes. Ainsi, et par rapport à cette inapplicabilité de la norme fondamentale de l'éthique de la discussion dont Dussel s'est servi pour indiquer la différence de point de départ entre ces deux éthiques, Apel souligne à plusieurs reprises le fait que nous devons, dans les sociétés actuelles et en tant qu'individus responsables qui argumentent, «reconnaître la norme procédurale d'une communauté idéale de communication et reconnaître [en même temps] notre appartenance à la communauté réelle - qui s'est historicisée - de communication $»^{25}$, communauté dans laquelle on trouve effectivement les injustices et les asymétries que Dussel a révélées. De cette tension dialectique entre la norme fondamentale idéale et la réalité politique résulte, d'après Apel, une troisième norme, applicable au plan stratégique, qui consistera à « contribuer autant que faire se peut à une transformation à long terme des rapports: transformation visant à atténuer la tension entre la norme idéale du règlement des conflits et la réalité politique et, de la sorte, à se rapprocher peu à peu des conditions idéales d'un règlement consensuel des conflits $»^{26}$. L'architectonique d'Apel se constitue donc de trois normes : la norme fondamentale, prioritaire et non stratégique d'une formation du consensus obtenu par la pure voie argumentative ; une deuxième norme de responsabilité qui assigne à l'homme politique le devoir de conserver et protéger la réalité qui lui a été confiée ; et une troisième norme, surgie de la tension entre les deux précédentes, qui vise à une modification des

(apellare), «affronter» ou «se trouver face à » quelqu'un avec qui s'établit une relation (inter). À différence du récriminer ou du reprocher, l' «interpeller », ou l' « interpellation », sont actifs : ils exigent une réparation, un changement. Voir Dussel, Apel, Ricoeur, Rorty y la filosofía de la Liberación, Guadalajara (México), ed. Universidad de Guadalajara, 1993, p. 57, N. 25 .

25. K.-O. Apel, Discussion et responsabilité, vol. 2, op. cit., p. 48.

26. Ibid., p. 49. 
circonstances réelles allant vers un rapprochement des conditions idéales permettant l'application de la norme fondamentale. Ce dernier principe de complémentarité montre dans quelle mesure Apel est conscient - contre les critiques de Dussel - de l'impossibilité d'une application immédiate du principe discursif.

En conséquence, si nous allons au-delà de l'énoncé de la norme fondamentale de l'éthique de la discussion et que nous tenons compte du «système de trois normes » que propose l'auteur, nous retrouvons chez Apel la considération des mêmes trois moments dont parlait le représentant de la philosophie de la libération : a) un moment de validité purement formel concernant la participation des affectés dans les discussions, b) un moment matériel qui, pour Dussel, se présente comme l'impossibilité de vivre de $80 \%$ de la population et pour Apel comme l'obligation de garantir la survie de l'humanité et donc de préserver les conditions de vie nécessaires à une humanité «qui continue dans les générations à venir », et c) un troisième moment, commun aussi aux deux auteurs, de transformation à long terme des rapports historiques dans le sens d'un progrès vers la réalisation de la dignité humaine et donc vers la réalisation progressive de la communauté idéale, libre d'injustices et de domination. Ce troisième principe est considéré chez ces deux auteurs comme une «idée régulatrice » de la raison pratique au sens kantien, c'est-à-dire, comme un principe normatif qui a « une force d'obligation lui permettant d'accomplir un devoir et de le guider dans la réalisation à long terme de ce qui s'approche d'un idéal $»^{27}$. Une idée régulatrice, à la différence d'une utopie, exprime l'idée que «rien dans le temps de l'expérience ne correspondra jamais pleinement à l'idéal visé $»^{28}$. La différence entre les deux auteurs apparaît dans l'ordre de ces trois moments. Si chez Apel la norme fondamentale est strictement formelle et doit être postérieurement appliquée empiriquement et historiquement, chez Dussel, c'est au contraire le principe matériel qui est antérieur, la norme formelle ayant pour fonction d'appliquer ce principe matériel.

\section{Responsabilité, a priori ou a posteriori ?}

Nous avons vu comment l'éthique de la libération de Dussel prend comme point de départ la prétendue inapplicabilité du principe fondamental de l'éthique de la discussion: la non-participation factuelle de $80 \%$ de la population $\mathrm{du}$ globe comme un type d'exclusion non-intentionnelle inévitable, c'est-à-dire la misère, l'oppression et l'exclusion des majorités. Face à ce constat empirique, la re-sponsabilité par Autrui nous oblige à «chercher dans le système ou Totalité en vigueur les causes de la victimisation, ce qui constitue le moment proprement critique de 
l'éthique $»^{29}$. Le moment premier de la transformation est le fait d'assumer la propre re-sponsabilité de la critique. Dussel écrit «re-sponsabilité » afin de distinguer sa propre conception de la responsabilité d'influence lévinasienne de celle a posteriori que l'auteur attribue à Hans Jonas mais aussi à Apel entre autres ${ }^{30}$. En effet, Dussel insiste à plusieurs reprises sur le fait que la re-sponsabilité dont il parle n'est pas une responsabilité weberienne a posteriori sur les effets de nos actes mais une re-sponsabilité au sens lévinasien : être responsables «par Autrui » par-devant le tribunal (de la communauté réelle ou en vigueur) afin de le libérer de son oppresseur. La re-sponsabilité se joue dès lors comme «critique » et «transformation » des causes dans le système qui provoque la misère, la domination et l'exclusion dont souffrent les victimes. Elle est aussi la condition pratique de la possibilité de tout argumenter, ce qui permet à Autrui de faire partie d'une «nouvelle» communauté argumentative et dès lors d'avancer vers un universalisme plus authentique. Cette re-sponsabilité est antérieure à toute argumentation discursive et en ce sens elle est a priori, antérieure à la responsabilité a posteriori des conséquences pour les «affectés» des décisions accordées dans les discussions. Mais l'éthique de la discussion d'Apel est-elle une simple éthique de la responsabilité a posteriori comparable à celle de Jonas ?

En effet, bien qu'Apel parle plus souvent de l'urgence pratique d'une macroéthique universaliste face à la nouvelle question d'une crise environnementale liée aux conséquences planétaires des activités collectives à l'ère de la science et de la technique, il reconnaît aussi dans certains passages, qu'à côté de ce problème global, il y a aussi celui d'une justice à l'échelle planétaire, d'un processus de progrès vers l'émancipation sociale «qui va de pair avec cette éthique». Comme nous l'avons vu, cette obligation de progrès est en même temps une obligation de transformer avant tout les rapports réels afin que la norme fondamentale de l'éthique de la discussion puisse être appliquée dans un futur.

Ainsi, dans le premier chapitre du deuxième volume de Discussion et responsabilité, Apel introduit son «système de trois normes » en réponse à la thèse principale de l'ouvrage Le Principe Responsabilité de Hans Jonas selon laquelle dans la situation de crise qu'on trouve dans les sociétés industrialisées des années quatre-vingt du siècle dernier, on doit abandonner toute utopie du progrès. En effet, selon Jonas, ce qui s'impose dans les sociétés des années quatre-vingt - sociétés qui se trouvent confrontées à un type nouveau de conflit lié à la crise environnementale dont on commence à être conscient à cette époque - c'est une «éthique de la conservation, une éthique de la préservation, de l'empêchement et non du progrès et du

29. E. Dussel, Ética de la Liberación, op .cit., p. 367-368. La traduction est nôtre.

30. Nous n'entrerons pas dans cet article sur la question de la pertinence de la considération de la responsabilité chez Hans Jonas comme une simple responsabilité a posteriori. Nous allons ici nous limiter à la lecture de Hans Jonas qui apparaît dans les textes d'E. Dussel et de K.-O. Apel. 
perfectionnement $»^{31}$. Face à cette affirmation, Apel considère que pour sauver l'existence et la dignité humaines, il ne suffit pas de la pure et simple préservation des choses et, dès lors, d'une simple responsabilité a posteriori des conséquences des activités collectives. Au contraire, et si on veut «créer à l'échelle planétaire des rapports sociaux conformes à la dignité humaine $»^{32}$, il est nécessaire de considérer en même temps une idée régulatrice du progrès technologique et du progrès social, c'est-à-dire que «dans la communauté réelle de communication qui constitue l'humanité présente, la communauté idéale de communication est toujours en instance de devoir être - progressivement - réalisée ${ }^{33}$. Apel introduit alors son troisième principe, applicable au plan stratégique, et «qui consistera à contribuer autant que faire se peut à une transformation à long terme des rapports : transformation visant à atténuer la tension entre la norme idéale du règlement des conflits et la réalité politique et, de la sorte, à se rapprocher peu à peu des conditions idéales d'un règlement consensuel des conflits $»^{34}$. Ce troisième principe est dès lors un principe de progrès et d'émancipation sociale qui agit comme une idée régulatrice visant la réalisation, sans jamais vraiment l'atteindre, des conditions sociales et politiques d'une communauté idéale et donc de la justice à l'échelle planétaire. Apel échappe ainsi en même temps aux possibles solutions sociales-darwinistes à la crise écologique qui affirment que, «d'un point de vue strictement biologique, la survie de l'humanité, dans la situation contemporaine où règnent la surpopulation et la pénurie des ressources, pourrait tout à fait être assurée en affamant certaines parties de la population, dans le tiers monde par exemple $»^{35}$. Ce type de réponse est incompatible avec une éthique universaliste qui tende à la réalisation de la justice à l'échelle planétaire, comme celle d'Apel.

La médiation chez Apel entre la rationalité éthique et la rationalité stratégique constitue l'élément nouveau, propre à l'éthique de la discussion, qui la distinguera de toutes les formes traditionnelles d' «éthique de la conviction». En même temps, cette médiation constitue une nouvelle proposition d'une éthique de la responsabilité solidaire. Grâce à ce troisième principe, l'éthique de la discussion peut se révéler être une éthique de la responsabilité collective de solidarité vis-à-vis des actions collectives tout en mettant l'accent sur ce qui la distingue du Principe Responsabilité chez Jonas. De plus, en ce qui concerne le débat d'Apel avec la philosophie de la libération, l'éthique de la discussion, précisée dans son «système de trois normes », peut ainsi se présenter comme une éthique non seulement de la responsabilité a posteriori des conséquences des accords, ce qu'elle reconnaît tout de même, mais aussi, et à la différence de Jonas, comme

31. H. Jonas, Le Principe Responsabilité, Paris, Cerf, 1990, p. 195 et suiv.

32. K.-O. Apel, Discussion et responsabilité, vol. 2, op. cit., p. 13.

33. Ibid., p. 28.

34. Ibid., p. 49.

35. Ibid., p. 13. 
une éthique de la responsabilité transcendantale, c'est-à-dire de la (co-) responsabilité comme "pré-condition transcendantale de la communauté humaine et de l'interaction réciproque ${ }^{36}$. Dès lors, chez Apel la responsabilité par les conséquences est orientée par deux critères :

a) une condition restrictive de préservation et garantie de la survie de l'humanité, au sens de Jonas,

b) l'idée régulatrice de progrès ou «objectif lointain de la réalisation approximative des relations de la communauté idéale de communication ${ }^{37}$.

Ce dernier critère semble aller dans le sens de la responsabilité a priori de Dussel, une responsabilité dont l'objectif est de transformer la «communauté réelle» et de construire une nouvelle communauté future dans laquelle la victime puisse participer - dans les mots de Dussel - et où la norme fondamentale de l'éthique de la discussion puisse être appliquée de sorte que la résolution des conflits se fasse par la formation d'un consensus entre toutes les parties concernées, lui-même atteint par la pure voie de la discussion - dans les mots d'Apel. En conséquence, il nous semble que finalement l'éthique de la discussion et l'éthique de la libération, malgré leurs différences, soutiennent des affirmations très proches, tout en accentuant des aspects différents dépendants de leurs inquiétudes fondamentales. Nous n'entrerons pas ici davantage dans ce sujet, étant donné qu'il fait l'objet de la partie ultime et suivante de ce travail.

\section{Conclusion : À propos d'une possible complémentarité entre l'éthique de la discussion et l'éthique de la libération}

$\mathrm{Au}$ long des différentes interventions qui constituent le dialogue entre l'éthique de la libération et l'éthique de la discussion, nos deux auteurs ont oscillé dans leur manière de situer leur éthique l'une par rapport à l'autre. La position qui reste pourtant la plus défendue quand on lit ces deux auteurs est celle selon laquelle, aux yeux d'Apel, les demandes de l'éthique de la libération constituent un cas concret de sa partie $B$ de l'éthique de la discussion, et symétriquement, d'après Dussel c'est l'éthique de la discussion qui représente un moment de son éthique de la libération. Néanmoins, il nous semble que, malgré les divergences méthodologiques issues de points de départ différents, on peut finalement affirmer une complémentarité réciproque, mutuellement enrichissante bien qu'en même temps conflictuelle, comme les propres auteurs le reconnaissent à plusieurs reprises.

36. K.-O. Apel, E. Dussel, Ética del discurso y ética de la liberación, op. cit., p. 135.

37. Ibid., p. 264. 
En effet, depuis son intervention lors de la première rencontre célébrée à Freiburg le 25 novembre 1989, Dussel se situe «avec Apel, au-delà d'Apel ». Il reconnaît l'importance de la démarche apelienne et de la découverte de la «communauté de communication» pour surpasser le solipsisme moderne. Néanmoins, la pensée d'Apel est aux yeux de Dussel insuffisante dans une situation de domination et de négation d'Autrui. Quand l'expérience de l'exclusion est un point de départ quotidien, il devient nécessaire de lui trouver un «lieu» philosophique, nous dira Dussel. L'auteur propose alors un élargissement de la définition de la communauté de communication apelienne en y incluant explicitement, comme un moment de la définition, l'extériorité virtuelle de chaque personne, d'Autrui que Dussel caractérise de «dis-tinct» et non simplement de «di-fférent ${ }^{38}$. L'éthique de la libération part ainsi de la proposition discursive, tout en la transformant afin de pouvoir finalement articuler le principe formel d'Apel avec le principe matériel. En conséquence, au-delà de la pragmatique transcendantale apelienne Dussel affirme l'importance de développer, pour une philosophie qui part d'Autrui en tant que pauvre, une «économique » ${ }^{39}$ qui pose l'obligation de participer à la «reproduction de la vie». La «communauté de producteurs» ou «communauté d'êtres vivants» est antérieure et, en ce sens, elle est la condition première du sujet argumentant. De même, l'irruption d'Autrui qui met en question l'universalisme totalitaire d'une communauté réelle de communication, celle-ci imposant en tant que dominateur sa propre particularité comme hégémonique et idéale, est condition de possibilité de toute nouvelle argumentation. En ce sens, l'interpellation, mais aussi la re-sponsabilité, sont, d'après le philosophe de l'éthique de la libération, également antérieures à l'argumentation. En conséquence, le principe fondamental de l'éthique de la discussion devient un moment secondaire dans l'architectonique de l'éthique de la libération : l'éthique de la discussion est prise pour un moment de l'éthique de la libération.

De manière symétrique, Apel reconnaît que l'éthique de la libération constitue un défi pour son éthique de la discussion mais il affirme en même temps qu'il s'agit d'un défi qui fait partie de la partie $B$ de son éthique, c'est-à-dire, de la partie de l'éthique qui traite de ces situations dans lesquelles les conditions pour l'application de la partie A de l'éthique de la discussion ne sont pas encore réalisées dans ce monde. En conséquence, l'exclusion constitue une question propre à cette partie $B$ et, dès lors la problématique que présente Dussel peut être résolue à l'intérieur de l'éthique de la discussion. Étant donné ces situations dans lesquelles on ne peut pas

\footnotetext{
38. Dussel distingue la «Di-fférence», définie à partir de l' «Identité », interne à la «totalité », et la «Dis-tinction » d'Autrui, c'est-à-dire, dès l' «Extériorité », ce qui représente une réelle altérité.

39. Pour l'éthique de la libération, qui part du fait massif de la misère et de l'impossibilité chez les majorités de reproduire la vie, il est essentiel de développer une «économique ». La relation économique est une relation «éthique-technologique » ou "pratico-poiétique », et non seulement productive.
} 
appliquer directement la norme fondamentale de la partie $A$ de l'éthique de la discussion, on doit alors s'acquitter du principe complémentaire de la partie $B$ de l'éthique. Ce principe est un principe régulateur de responsabilité qui contribue à une politique à long terme de changement de la situation globale dans le monde. Dès lors, c'est un principe qui offre «dans la partie $B$ de l'éthique de la discussion une possible justification pour toute stratégie de «libération» possible dans le sens d'une réalisation progressive des conditions d'application de la partie A de l'éthique de la discussion $»^{40}$. De ce fait, l'interpellation des exclus de la discussion articulée par Dussel ne met pas en question, d'après Apel, l'approche pragmatique et transcendantale de l'éthique de la discussion; il s'agit au contraire d'un «problème caractéristique de la partie $B$ de l'éthique ».

Néanmoins, l'exclusion dont parle Dussel n'est pas seulement une exclusion de la discussion, mais également une exclusion de la vie: l'impossibilité de «produire, reproduire et développer» la vie. Apel reconnaît l'importance de la vie comme référence ultime, par exemple quand il parle de la surpopulation, de la faim ou de la destruction écologique comme des menaces contre la vie humaine. Mais, à la différence de la philosophie de la libération, il ne situe pas cette question comme un point de départ. En effet, bien qu'Apel reconnaisse que l'interpellation des exclus constitue un défi pour l'éthique de la discussion puisqu'elle constitue un apport innovateur à la discussion illimitée de la communauté idéale de communication, il considère cependant qu'une «fondation au sens strict à partir de la rencontre avec Autrui ${ }^{41}$ n'est pas possible. Ainsi, lors de la sixième rencontre célébrée à Eichstätt en avril 1995, le philosophe de la discussion écrit : «À mon avis, l'on ne peut pas comparer et confronter l'existence corporelle à la discussion transcendantale de la philosophie ; puisque, pour l'exprimer de manière emphatique, l'on peut réfléchir ou effectuer une discussion valide ou invalide sur la vie humaine et ses conditions, mais l'on ne peut pas vivre sur la pensée ou la discussion. Cette expérience linguistique montre que ce qui est indépassable au sens transcendantal ne peut pas être la vie ou l'existence corporelle ${ }^{42}$, même si elle est fondamentale du point de vue ontologique ou anthropologique.

Toutefois, le philosophe de la libération nie que son éthique puisse être réduite à un cas précis de la partie $B$, c'est-à-dire d'application de l'éthique de la discussion. Ainsi, Dussel affirme à plusieurs reprises que l'a priori de la re-connaissance d'Autrui est pré-scientifique et pré-réflexif, et en ce sens, il est antérieur à la fondation de l'éthique et à toute argumentation possible. Dussel insiste également sur le fait qu'il ne nie pas la fondation mais sa priorité, car pour l'éthique de la libération, comme nous l'avons vu, le critère et point de départ est la corporalité souffrante du dominé ou de l'exclu: l'altérité d'Autrui. D'après Dussel, ce n'est pas une question d'application

40. K.-O. Apel, E. Dussel, Ética del discurso y ética de la liberación, op. cit., p. 332.

41. Ibid., p. 35 .

42. Ibid., p. 326. 
de la norme fondamentale, comme c'était le cas pour l'éthique de la discussion. Au contraire, il inverse l'ordre, en reprenant les acquis de l'éthique de la discussion, pour conclure qu'en réalité c'est la norme formelle qui a pour fonction d'appliquer le principe matériel, qui lui est antérieur.

En lisant les articles correspondants aux successives interventions qui constituent le dialogue entre l'éthique de la discussion et l'éthique de la libération, nous arrivons à la conclusion que chacune de ces deux éthiques représente un défi pour l'autre et que, dès lors, une complémentarité est possible : nous pouvons affirmer en résumé que «l'éthique de la libération et l'éthique de la discussion a priori se nécessitent l'une l'autre », comme l'affirmait Apel lors de la quatrième rencontre. Nous constatons également que cette possible complémentarité, bien qu'inévitablement conflictuelle en raison d'énormes divergences méthodologiques, est sans doute en même temps mutuellement enrichissante. D'autre part, le dialogue entre l'éthique de la discussion et l'éthique de la libération détient un intérêt particulier car il représente une initiative en faveur du dialogue entre la philosophie européenne et la philosophie des périphéries du monde actuel (l'Amérique latine, l'Afrique et l'Asie), et en ce sens, elle constitue un cas concret dans le domaine de la philosophie du dialogue Nord-Sud que nous estimons, de nos jours, absolument nécessaire. 